\title{
ASSESSMENT OF MACULAR FUNCTION BY FOCAL ELECTRORETINOGRAM AND PATTERN ELECTRORETINOGRAM BEFORE AND AFTER EPIMACULAR MEMBRANE SURGERY
}

\author{
VINCENZO PARISI, MD, ANDREA MARIA COPPÈ, MD, \\ GELTRUDE GALLINARO, BSE, MARIO STIRPE, MD
}

\begin{abstract}
Purpose: To evaluate macular function before and after surgical peeling of idiopathic epimacular membrane (EMM).

Methods: Logarithm of the minimal angle of resolution visual acuity and results of focal (central $9 \times 9^{\circ}$ ) electroretinogram (fERG), pattern electroretinogram ( $\left.p E R G\right)$, and optical coherence tomography (OCT) assessment of macular volume were evaluated for 22 eyes of 22 patients (mean age \pm SD, $63.20 \pm 10.0$ years) with EMM preoperatively (baseline) and 6 months after surgical peeling. Preoperative visual acuity and fERG and pERG amplitudes observed in EMM eyes were compared with those in 15 age-matched control eyes.
\end{abstract}

Results: In the preoperative evaluation, EMM eyes had a significant $(P<0.01$; one-way analysis of variance) reduction in visual acuity and fERG and pERG amplitudes and an increase in OCT macular volume when compared with controls. In EMM eyes, the decrease in visual acuity was significantly correlated $(P<0.01$, Pearson test) to the reduction in fERG and pERG amplitudes. At the postoperative evaluation, EMM eyes had a correlated significant $(P<0.01)$ increase in visual acuity, fERG amplitude, and pERG amplitude with respect to the preoperative values. All EMM eyes had a significant $(P<0.01)$ reduction in macular volume, and retinal microanatomy was restored to normal conditions.

Conclusion: In EMM eyes, the decrease in visual acuity is related to dysfunction of both preganglionic (abnormal fERG) and ganglionic (abnormal pERG) macular elements. Surgical removal of EMM may induce improvement of the function of both outer and innermost macular retinal layers, leading to a related increase in visual acuity.

RETINA 27:312-320, 2007

$\mathrm{E}$ yes with idiopathic epimacular membrane (EMM) have an increase in macular thickness that can be revealed by optical coherence tomography $(\mathrm{OCT})^{1-3}$

From Fondazione G.B. Bietti, IRCCS, Rome, Italy.

The authors have no proprietary interest in the development or marketing of the instruments used.

Reprint requests: Vincenzo Parisi, MD, Fondazione per l'Oftalmologia G.B. Bietti, IRCCS, Via Santa Maria Goretti, 66 00199 Rome, Italy; e-mail: vparisi@tin.it and a macular dysfunction identified by subjective $e^{4,5}$ or electrophysiological (i.e., focal electroretinogram [fERG] and multifocal electroretinogram ${ }^{6-9}$ ) tests of visual function. Nevertheless, because fERG and multifocal electroretinogram originate from preganglionic elements, ${ }^{6,7-16}$ these electrophysiological tests do not allow the objective functional evaluation of the innermost retinal layers of the macular region. Therefore, the function of ganglion cells and their fibers, which 
can be specifically assessed by pattern electroretinogram (pERG), ${ }^{6,15,17-19}$ in eyes with EMM is at present unknown.

The aim of the current study was to evaluate the function of the outer and innermost retinal layers of the macular region before and after surgical peeling of idiopathic EMM by means of fERG and pERG.

\section{Materials and Methods}

\section{Patients}

Twenty-two eyes of 22 patients (8 men and 14 women; mean age $\pm \mathrm{SD}, 63.20 \pm 10.0$ years) with EMM were enrolled in the study (22 EMM eyes). The clinical diagnosis of EMM was based on results of indirect binocular ophthalmoscopy with a +90-diopter no-contact lens (Volk Optical, Inc., OH) and OCT (see below), which revealed an increase in macular volume associated with optically hyperreflective epiretinal echoes due to the presence of a membrane. Because several conditions may induce changes in fERG and pERG responses ${ }^{6}$ and their presence may represent a potential confounding factor in the present study, enrolled patients were selected from a larger number of patients (52 patients) with EMM, on the basis of the following exclusion criteria: presence of dense lens opacities, ocular hypertension or glaucoma, diabetic retinopathy, drusen and age-related macular disease, and other general diseases, such as systemic hypertension, diabetes, and connective tissue or neurologic diseases. All EMM eyes enrolled in the study were phakic with a refractive error of \pm 0.50 spherical diopter.

EMM eyes were compared with 15 eyes of 15 age-matched (mean age $\pm \mathrm{SD}, 62.03 \pm 11.05$ years) control subjects ( 15 control eyes). The same exclusion criteria used for EMM patients were applied to control subjects. In control eyes, the refractive error was \pm 0.50 spherical diopter.

Visual acuity, fERG, pERG, and OCT findings were assessed for each control subject and each EMM patient at baseline using the following methods.

\section{Best-Corrected Visual Acuity}

Best-corrected visual acuity was assessed by the Modified ETDRS Table (Lighthouse, Low Vision Products, Long Island, NY). Visual acuity (expressed in logarithm of the minimal angle of resolution) was determined at distances of $4 \mathrm{~m}, 2 \mathrm{~m}, 1 \mathrm{~m}$, and $0.5 \mathrm{~m}$.

\section{fERG and $p E R G$}

fERG and pERG were performed according to a previously reported method. ${ }^{6,14,15,19}$ Briefly, fERG was performed in response to a $9 \times 9^{\circ}$ uniform field, flickered sinusoidally at $30 \mathrm{~Hz}$ (mean luminance, 80 $\mathrm{cd} / \mathrm{m}^{2}$; modulation depth, 93.5\%), ${ }^{14,15}$ and $\mathrm{pERG}$ was performed in response to sinusoidal gratings of 2 cycles $/{ }^{\circ}$ spatial frequency with $90 \%$ contrast, stimulating the central $9 \times 9^{\circ}$ generated in the television monitor (mean luminance, $80 \mathrm{~cd} / \mathrm{m}^{2}$ ) and modulated in counterphase at $8 \mathrm{~Hz} \cdot{ }^{15,19}$ Acquired signals underwent Fourier analysis to isolate the fundamental harmonic component (fERG, temporal frequency of 30 $\mathrm{Hz}$ [first harmonic (1F)]; pERG, temporal frequency of $8 \mathrm{~Hz}$ [second harmonic (2P)]), whose amplitude $(\mu \mathrm{V})$ and phase $\left(^{\circ}\right)$ were measured.

\section{$O C T$}

OCT was performed by commercially available instrumentation (OCT3 Stratus; Carl Zeiss Ophthalmic Systems, Inc., Humphrey Division, Dublin, CA). A superluminescent diode $(820 \mathrm{~nm})$ was used to scan and analyze the macular area. After pupillary dilation, OCT images of the macular region were obtained with horizontal and vertical scans, with a length of $6.5 \mathrm{~mm}$. The axial resolution was $<10 \mu \mathrm{m}$, and the transverse resolution was $20 \mu \mathrm{m}$. Macular morphology was analyzed using the software provided with the instrument (OCT3 Software Version 2.0) and the scan acquisition protocol Fast Macula that analyzes the macular region with six consecutive radial scans (radius, $3 \mathrm{~mm}$ ) centered with respect to the fixation point; the same software provides measurement of the mean macular volume $\left(\mathrm{mm}^{3}\right)$. Preoperatively, all control eyes and EMM eyes underwent at least two OCT examinations 1 day to 7 days apart, to determine test-retest variability.

\section{Surgical Procedure}

According to other studies, ${ }^{20-22}$ the surgical procedure consisted of standard three-port pars plana vitrectomy with complete removal of EMM by retinal forceps without intentional peeling of internal limiting membrane (ILM). Although we did not perform histopathological examinations, it is possible that in some cases ILM may have been unintentionally peeled off together with EMM. No silicone oil and/or intraocular lens was introduced after vitrectomy in any of the eyes enrolled.

\section{Follow-Up}

For EMM eyes, visual acuity, fERG, pERG, and OCT findings were assessed 6 months (mean $\pm \mathrm{SD}$, $180 \pm 5$ days) after surgical treatment. The research 
Table 1. Clinical Characteristics of EMM Eyes Observed at Baseline and 6 Months After Surgical Treatment

\begin{tabular}{|c|c|c|c|c|c|c|c|c|c|c|}
\hline \multirow[b]{2}{*}{ Patient } & \multirow[b]{2}{*}{ Age (y) } & \multicolumn{2}{|c|}{$\begin{array}{l}\text { Visual Acuity } \\
\text { (logMAR) }\end{array}$} & \multirow{2}{*}{$\begin{array}{l}\text { Surgical } \\
\text { Treatment }\end{array}$} & \multicolumn{2}{|c|}{$\begin{array}{c}\text { fERG 1F } \\
\text { Amplitude }(\mu \mathrm{V})\end{array}$} & \multicolumn{2}{|c|}{$\begin{array}{c}\text { pERG 2P } \\
\text { Amplitude }(\mu \mathrm{V})\end{array}$} & \multicolumn{2}{|c|}{$\begin{array}{l}\text { OCT Macular } \\
\text { Volume }\left(\mathrm{mm}^{3}\right)\end{array}$} \\
\hline & & Baseline & $6 \mathrm{mo}$ & & Baseline & $6 \mathrm{mo}$ & Baseline & $6 \mathrm{mo}$ & Baseline & $6 \mathrm{mo}$ \\
\hline 1 & 64 & 1.0 & 0.5 & PPV & 0.42 & 0.64 & 0.33 & 0.62 & 12.14 & 10.23 \\
\hline 2 & 59 & 0.3 & 0.15 & PPV + air & 0.72 & 0.96 & 0.48 & 0.73 & 8.71 & 7.52 \\
\hline 3 & 69 & 0.5 & 0.2 & PPV & 0.43 & 0.83 & 0.42 & 0.67 & 8.57 & 8.02 \\
\hline 4 & 72 & 0.5 & 0.2 & PPV & 0.65 & 0.88 & 0.46 & 0.71 & 9.07 & 8.25 \\
\hline 5 & 69 & 0.3 & 0.3 & PPV + gas & 0.53 & 0.74 & 0.58 & 0.83 & 8.49 & 8.12 \\
\hline 6 & 67 & 1.0 & 0.5 & PPV + gas & 0.32 & 0.55 & 0.39 & 0.62 & 10.76 & 9.31 \\
\hline 7 & 75 & 0.8 & 0.2 & $\mathrm{PPV}+$ gas & 0.43 & 0.92 & 0.63 & 1.02 & 10.47 & 9.98 \\
\hline 8 & 33 & 1.2 & 0.7 & $\mathrm{PPV}+$ air & 0.25 & 0.55 & 0.22 & 0.46 & 13.81 & 9.73 \\
\hline 9 & 54 & 0.8 & 0.1 & PPV & 0.52 & 1.21 & 0.34 & 0.68 & 9.59 & 8.91 \\
\hline 10 & 62 & 0.4 & 0.2 & PPV & 0.45 & 0.74 & 0.44 & 0.82 & 8.65 & 8.23 \\
\hline 11 & 67 & 0.4 & 0.4 & PPV & 0.44 & 0.45 & 0.72 & 0.74 & 8.96 & 8.24 \\
\hline 12 & 66 & 0.3 & 0.1 & PPV & 0.66 & 0.94 & 0.62 & 0.85 & 8.62 & 8.11 \\
\hline 13 & 72 & 0.4 & 0.4 & PPV + air & 0.53 & 0.54 & 0.62 & 0.64 & 8.42 & 7.94 \\
\hline 14 & 42 & 0.8 & 0.5 & $\mathrm{PPV}+$ gas & 0.62 & 0.92 & 0.43 & 0.66 & 8.96 & 7.81 \\
\hline 15 & 76 & 0.8 & 0.7 & PPV + air & 0.49 & 0.71 & 0.54 & 0.53 & 8.76 & 8.12 \\
\hline 16 & 64 & 0.3 & 0.1 & PPV & 1.12 & 1.23 & 0.76 & 0.93 & 8.58 & 7.43 \\
\hline 17 & 67 & 0.4 & 0.1 & PPV & 1.06 & 1.32 & 0.72 & 0.97 & 8.13 & 7.43 \\
\hline 18 & 59 & 0.3 & 0.0 & PPV & 1.04 & 1.24 & 0.68 & 1.04 & 8.29 & 7.21 \\
\hline 19 & 58 & 0.2 & 0.0 & PPV & 1.09 & 1.36 & 0.78 & 1.12 & 8.38 & 7.49 \\
\hline 20 & 63 & 0.4 & 0.1 & PPV & 1.06 & 1.29 & 0.80 & 1.09 & 9.42 & 8.57 \\
\hline 21 & 64 & 0.3 & 0.1 & PPV & 1.15 & 1.35 & 0.67 & 0.94 & 8.49 & 8.12 \\
\hline 22 & 68 & 0.3 & 0.0 & PPV & 1.19 & 1.42 & 0.77 & 1.16 & 8.41 & 7.36 \\
\hline
\end{tabular}

EMM, epimacular membrane; logMAR, logarithm of the minimal angle of resolution; fERG, focal electroretinogram; $1 F$, first harmonic; pERG, pattern electroretinogram; 2P, second harmonic; OCT, optical coherence tomography; PPV, standard three-port pars plana vitrectomy.

followed the tenets of the Declaration of Helsinki, and the study was approved by the local ethics committee.

\section{Statistic Analysis}

Normal limits were obtained for control subjects by calculating mean values - 3 SDs for fERG and pERG amplitudes and mean values +3 SDs for OCT macular volume. Test-retest data for fERG, pERG, and OCT results were expressed as the mean difference between two records obtained in separate sessions \pm SD of this difference; $95 \%$ confidence limits of testretest variability for normal subjects and patients were established assuming a normal distribution. Taking into account this test-retest variability, in all control and EMM eyes, fERG and pERG recordings and OCT macular volume with the smallest values were selected, and the corresponding values were considered in the statistical analysis.

Results for control and EMM eyes observed at baseline were compared by one-way analysis of variance. Changes in functional (visual acuity, fERG, and pERG) and morphologic (OCT) parameters observed in EMM eyes 6 months after surgical treatment were compared with baseline (pretreatment) values by oneway analysis of variance. Pearson correlation was used to correlate visual function parameters (visual acuity and fERG $1 \mathrm{~F}$ and pERG $2 \mathrm{P} \log$ amplitudes) observed at baseline and at follow-up. fERG and pERG amplitudes underwent logarithmic transformation to better approximate a normal distribution.

In all analyses, $P<0.01$ was considered statistically significant.

\section{Results}

\section{Baseline}

The clinical characteristics of EMM eyes observed at baseline are reported in Table 1. Examples of fERG and pERG recordings are shown in Figure 1. Examples of OCT results are shown in Figure 2. Mean data $\pm \mathrm{SD}$ are presented in Table 2 .

Test-retest data for fERG amplitudes were as follows: control eyes-mean $\pm \mathrm{SD}, 0.0065 \pm 0.098 \mu \mathrm{V}$ (95\% confidence limits, -0.189 to $0.202 \mu \mathrm{V}$ ); EMM eyes-mean $\pm \mathrm{SD}, 0.0072 \pm 0.099 \mu \mathrm{V}(95 \%$ confidence limits, -0.190 to $0.205 \mu \mathrm{V})$. Test-retest data for pERG amplitudes were as follows: control eyesmean $\pm \mathrm{SD}, 0.0087 \pm 0.096 \mu \mathrm{V}(95 \%$ confidence limits, -0.183 to $0.200 \mu \mathrm{V})$; EMM eyes-mean $\pm \mathrm{SD}$, $0.013 \pm 0.097 \mu \mathrm{V}(95 \%$ confidence limits, -0.181 to 


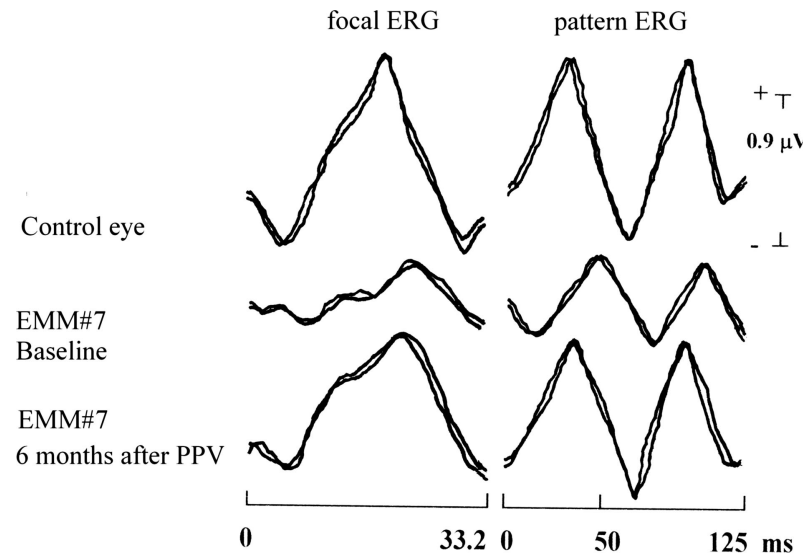

Fig. 1. Examples of focal electroretinogram (focal ERG) and pattern electroretinogram (pattern ERG) recordings for one control eye and one epimacular membrane (EMM) eye (EMM\#7) preoperatively (baseline) and 6 months after surgical removal of EMM by pars plana vitrectomy (PPV). At baseline, EMM\#7 had focal ERG and pattern ERG responses with reduced amplitudes and delayed phases with respect to the control eye. Six months after surgical treatment, EMM\#7 had increased amplitudes and shortened phases of focal ERG and pattern ERG responses with respect to baseline recordings.

$0.207 \mu \mathrm{V})$. Test-retest data for OCT macular volume were as follows: control eyes-mean $\pm \mathrm{SD}, 0.0216 \pm$ $0.0816 \mathrm{~mm}^{3}$ (95\% confidence limits, 0.146 to 0.1848 $\mathrm{mm}^{3}$ ); EMM eyes-mean $\pm \mathrm{SD}, 0.0236 \pm 0.0917$ $\mathrm{mm}^{3}\left(95 \%\right.$ confidence limits, 0.1598 to $\left.0.2070 \mathrm{~mm}^{3}\right)$.

All control eyes had visual acuity, fERG $1 \mathrm{~F}$ and pERG $2 \mathrm{P}$ amplitudes, fERG $1 \mathrm{~F}$ and pERG $2 \mathrm{P}$ phases, and OCT macular volume within our normal limits. In all EMM eyes, abnormal visual acuity, OCT macular volume, and pERG $2 \mathrm{P}$ amplitudes and phases were observed with respect to our normal limits (mean values for control eyes +3 SDs for pERG $2 \mathrm{P}$ phases and OCT macular volume; mean values for control eyes - 3 SDs for pERG 2P amplitudes).

In 7 EMM eyes, we observed fERG amplitudes and phases within our normal limits (mean values for control eyes - 3 SDs for fERG $1 F$ amplitudes; mean values for control eyes +3 SDs for fERG $1 \mathrm{~F}$ phases), while 15 EMM eyes had abnormal fERG amplitudes and phases. Therefore, in these 7 EMM eyes, concomitant OCT and pERG abnormalities and normal fERG results were found, while in $15 \mathrm{EMM}$ eyes, abnormal OCT, fERG, and pERG findings were detected.

In Figure 3, individual fERG $1 \mathrm{~F}$ amplitudes and pERG 2P amplitudes observed in EMM eyes are plotted as a function of the corresponding visual acuities. Significant correlations $(P<0.01)$ were observed between visual acuity and these electrophysiological parameters of macular function. On average, EMM eyes had significantly reduced $(P<0.01)$ visual acuity and fERG $1 \mathrm{~F}$ and $\mathrm{pERG} 2 \mathrm{P}$ amplitudes, significantly delayed $(P<0.01)$ fERG $1 \mathrm{~F}$ and pERG $2 \mathrm{P}$ phases, and significantly increased $(P<0.01)$ OCT macular volume when compared with controls.

OCT, in addition to quantitative analysis (e.g., measurement of the macular volume), revealed qualitative changes in retinal microanatomy in all EMM eyes. These changes, observed in all EMM eyes but with an interindividual variability, consisted of epiretinal echoes; these echoes represented tissue adherent to the macular retina that in several cases was not easy to differentiate from the inner retina (Fig. 2, EMM\#14 [left side]). In some cases, only a wrinkling of ILM could be observed (Fig. 2, EMM\#22). The underlying retina showed irregular and diffuse changes in optical density, related to different degrees of macular edema (Fig. 2, EMM\#1 and EMM\#14); in some cases, microcystic spaces corresponding to the region of the inner nuclear and inner plexiform layer could be detected (Fig. 2, EMM\#1). In all EMM eyes, distortion of the inner retinal elements expressed by an irregular optical density involving the innermost retinal layers was also detectable.

\section{Follow-Up: 6 Months After Surgical Treatment}

The clinical characteristics of EMM eyes observed at 6 months are reported in Table 1. Examples of fERG and pERG recordings are shown in Figure 1. Examples of OCT results are shown in Figure 2. Mean data \pm SD are presented in Table 2 .

Six months after surgical treatment, visual acuity was increased in 19 EMM eyes and unmodified in 3 EMM eyes with respect to baseline. Seventeen EMM eyes had increased fERG $1 \mathrm{~F}$ amplitudes (values exceeding the intraindividual variability resulting from test-retest analysis) and shortened fERG 1F phases. Focal ERG responses with unmodified amplitudes (values within intraindividual variability resulting from test-retest analysis) and phases were found in five EMM eyes. Eighteen EMM eyes had increased pERG 2P amplitudes (values exceeding the intraindividual variability resulting from test-retest analysis) and shortened pERG $2 \mathrm{P}$ phases, while in four EMM eyes, we observed $\mathrm{pERG}$ responses with unmodified amplitudes and phases (values within the intraindividual variability resulting from test-retest analysis).

A reduction in OCT macular volume (values exceeding the intraindividual variability resulting from testretest analysis) was found in all EMM eyes. On average, EMM eyes had a significant $(P<0.01)$ increase in visual acuity and in fERG $1 \mathrm{~F}$ and $\mathrm{pERG} 2 \mathrm{P}$ amplitudes, a significant shortening in fERG $1 \mathrm{~F}$ and $\mathrm{pERG} 2 \mathrm{P}$ phases, and a significant reduction in OCT macular volume, with respect to preoperative values. In Figure 4, differences in visual acuity observed in individual EMM eyes with respect to baseline values are plotted as a 

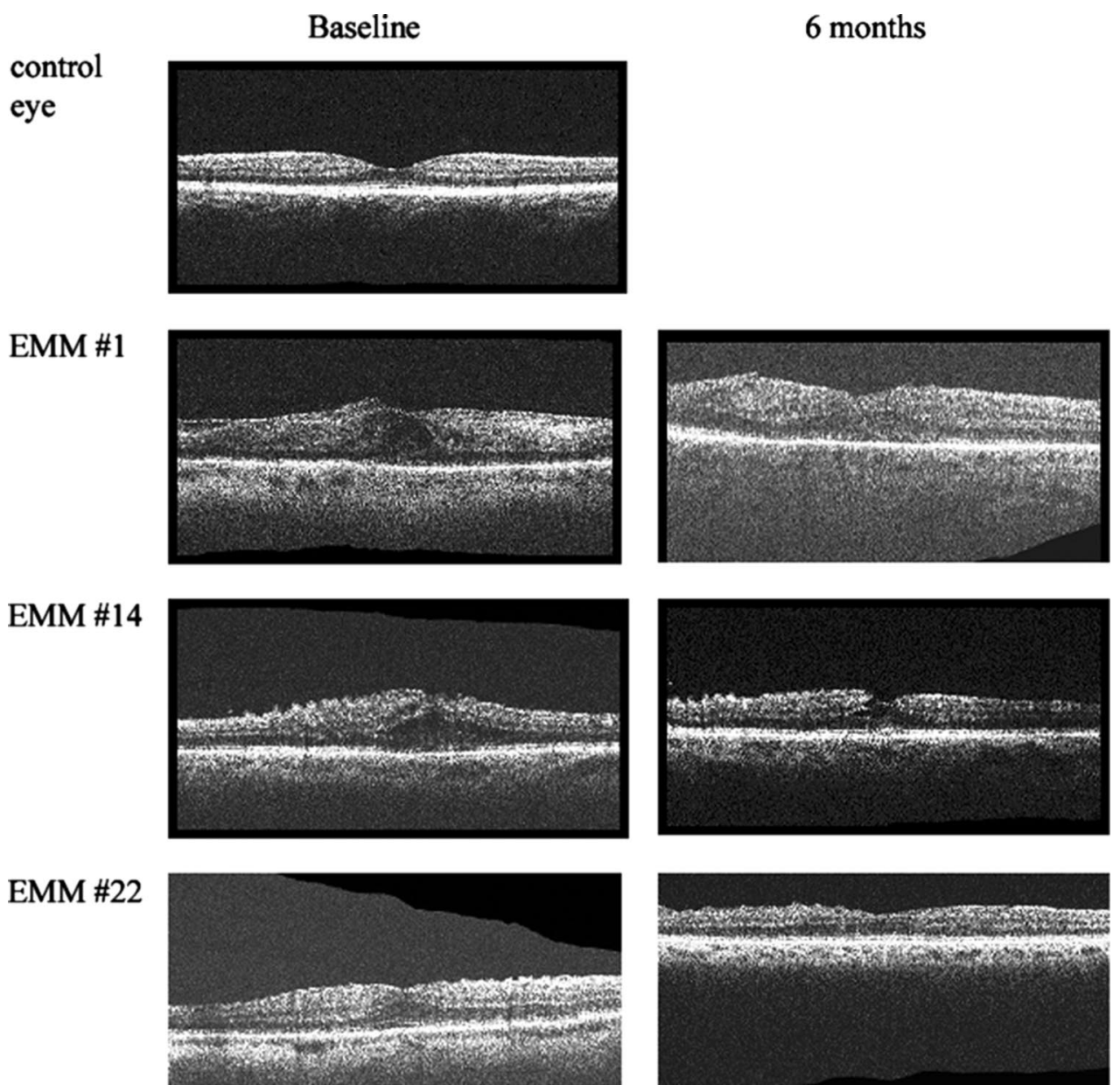

Fig. 2. Examples of optical coherence tomography (OCT) results for one control eye and three eyes epimacular membrane (EMM) eyes (EMM\#1, EMM\#14, and EMM\#22) preoperatively (baseline) and 6 months after surgical removal of EMM by pars plana vitrectomy (PPV). At baseline, EMM eyes had an increase in macular thickness associated with optically hyperreflective epiretinal echoes due to the presence of a membrane; cystic spaces were present within the inner plexiform layers in EMM\#1 and EMM\#14. In all EMM eyes, macular volume (EMM\#1, 12.14 mm³ ${ }^{3}$ EMM\#14, 8.96 $\mathrm{mm}^{3}$; EMM\#22, $8.41 \mathrm{~mm}^{3}$ ) was increased with respect to the normal value (mean $+3 \mathrm{SDs}, 7.45 \mathrm{~mm}^{3}$ ). Six months after surgical treatment, the hyperreflective epiretinal echoes disappeared almost completely, and a reduction of the macular thickening associated with a reduction in macular volume (EMM\#1, $10.43 \mathrm{~mm}^{3}$; EMM\#14, $7.81 \mathrm{~mm}^{3}$; EMM\#22, $7.36 \mathrm{~mm}^{3}$ ) was observed with respect to the preoperative values. In addition, the inner retinal microanatomy, which appeared altered at baseline, was almost completely restored to its normal structure 6 months after surgery. All OCT B-scans were converted into greyscale and tuned for lightness and contrast to enhance visualization of the retinal details.

function of the corresponding differences in fERG $1 \mathrm{~F}$ and pERG 2P amplitudes. Significant correlations $(P<$ $0.01)$ were observed between the differences in visual acuity and the differences in fERG $1 \mathrm{~F}$ and pERG $2 \mathrm{P}$ amplitudes.

Postoperative morphologic evaluation of the macular microanatomy in EMM eyes revealed several changes with respect to the preoperative condition. In all EMM eyes, the hyperreflective epiretinal echoes disappeared almost completely, and an overall reduction of macular thickening was observed associated with increased optical density (Fig. 2, EMM\#1). In addition, the retinal layers that appeared to be altered at baseline OCT showed diffuse recovery, restoring an almost normal retinal structure (Fig. 2, EMM\#14); distortion of the inner retinal elements expressed by irregular optical density in the region corresponding to the innermost retinal layers partially disappeared.

\section{Discussion}

The aim of our study was to evaluate, by fERG and pERG, the function of the outer and innermost retinal layers of the macular region in eyes with idiopathic EMM before (baseline) and 6 months after surgical peeling.

\section{Baseline}

At baseline, EMM eyes had a significant decrease in visual acuity and fERG $1 \mathrm{~F}$ and pERG $2 \mathrm{P}$ amplitudes when compared with control eyes; in addition, OCT revealed an increase in macular volume and 
Table 2. Mean \pm 1 SD of LogMAR Visual Acuity and Electrophysiological (Focal ERG and Pattern ERG) Parameters Observed in Control Eyes and EMM Eyes at Baseline and 6 Months After Surgical Treatment

\begin{tabular}{|c|c|c|c|}
\hline \multirow[b]{2}{*}{ Parameter } & \multirow{2}{*}{$\begin{array}{l}\text { Controls } \\
(\mathrm{n}=15)\end{array}$} & \multicolumn{2}{|c|}{ EMM Eyes $(n=22)$} \\
\hline & & Baseline & $6 \mathrm{mo}$ \\
\hline $\begin{array}{l}\text { Visual } \\
\text { acuity(logMAR) }\end{array}$ & $0.0 \pm 0.0$ & $\begin{array}{c}0.532 \pm 0.288 \\
\text { A vs } \mathrm{C}: \mathrm{f}(1,36)=50.72, P<0.001\end{array}$ & $\begin{array}{c}0.252 \pm 0.216 \\
\text { A vs } \mathrm{B}: \mathrm{f}(1,43)=13.31, P<0.001\end{array}$ \\
\hline $\begin{array}{l}\text { Focal ERG 1F } \\
\text { amplitude } \\
(\mu \mathrm{V})\end{array}$ & $1.370 \pm 0.119$ & $\begin{array}{c}0.690 \pm 0.308 \\
\text { A vs } \mathrm{C}: \mathrm{f}(1,36)=65.90, P<0.001\end{array}$ & A vs $B: \mathrm{f}(1,43)=7.51, P=0.009$ \\
\hline $\begin{array}{l}\text { Focal ERG 1F } \\
\text { phase }\left({ }^{\circ}\right)\end{array}$ & $-97.20 \pm 3.81$ & $\begin{array}{c}-146.8 \pm 11.23 \\
\text { A vs } \mathrm{C}: \mathrm{f}(1,36)=269.3, P<0.001\end{array}$ & $\begin{array}{c}-118.4 \pm 9.1 \\
\text { A vs } \mathrm{B}: \mathrm{f}(1,43)=84.93, P<0.001\end{array}$ \\
\hline $\begin{array}{l}\text { Pattern ERG } \\
2 \mathrm{P} \text { amplitude } \\
(\mu \mathrm{V})\end{array}$ & $1.310 \pm 0.164$ & $\begin{array}{c}0.564 \pm 0.168 \\
\text { A vs } \mathrm{C}: \mathrm{f}(1,36)=179.2, P<0.001\end{array}$ & $\begin{array}{c}0.810 \pm 0.199 \\
\text { A vs } B: \mathrm{f}(1,43)=19.63, P<0.001\end{array}$ \\
\hline $\begin{array}{l}\text { Pattern ERG } \\
2 P \text { phase }\left({ }^{\circ}\right)\end{array}$ & $-96.80 \pm 3.36$ & $\begin{array}{c}-137.4 \pm 12.31 \\
\text { A vs } \mathrm{C}: \mathrm{f}(1,36)=154.0, P<0.001\end{array}$ & $\begin{array}{c}-109.8 \pm 10.22 \\
\text { A vs } \mathrm{B}: \mathrm{f}(1,43)=65.47, P<0.001\end{array}$ \\
\hline $\begin{array}{l}\text { OCT macular } \\
\text { volume }\left(\mathrm{mm}^{3}\right)\end{array}$ & $7.06 \pm 0.13$ & $\begin{array}{c}9.26 \pm 1.396 \\
\text { A vs } \mathrm{C}: \mathrm{f}(1,36)=36.17, P<0.001\end{array}$ & $\begin{array}{c}8.28 \pm 0.859 \\
A \text { vs } B: \mathrm{f}(1,43)=7.86, P=0.0076\end{array}$ \\
\hline
\end{tabular}

Data observed for EMM eyes at baseline were compared with those for control eyes (C) by one-way analysis of variance (ANOVA [A]). The data observed for EMM eyes 6 months after surgical treatment were compared with those at baseline (B) by ANOVA.

LogMAR, logarithm of the minimal angle of resolution; ERG, electroretinogram; EMM, epimacular membrane; $n$, number of eyes enrolled in the study; $1 \mathrm{~F}$, first harmonic; $2 \mathrm{P}$, second harmonic; OCT, optical coherence tomography.

particular changes in macular microanatomy (see above). Visual acuity reduction observed in EMM eyes correlated to the electrophysiological abnormalities (Fig. 3), and associated morphologic retinal changes detectable by OCT may be explained by the events occurring in the macular structures in the presence of epiretinal membrane.

Histologic examinations suggested that several factors (such as the presence of incomplete posterior vitreous detachment or the presence of vitreomacular traction syndrome ${ }^{20}$ ) may induce cell proliferation in retinal ILM in eyes with EMM. Several cell types, such as astrocytes, fibrocytes, hyalocytes, macrophages, and retinal pigment epithelial cells, can be found in epiretinal membrane, ${ }^{21-24}$ and many of them have the ability to develop myofibroblast characteristics. $^{25}$ When this event occurs, contraction of the membrane may cause macular distortion, axoplasmic stasis, and macular edema of variable amount.

In our EMM eyes, the presence of macular edema was documented by OCT showing that an optically hyperdense epiretinal layer (the membrane) was generally associated with thickening and optical hypodensity in the underlying macular neuroretina; in more severe cases, cystic spaces were found within the region corresponding to the inner plexiform layer. Our finding is in agreement with those of other previous studies. ${ }^{1,26}$

The presence of abnormal fERG responses in our 15 EMM eyes suggests that functional impairment occurs in the outer retinal layers (preganglionic elements $)^{6-17}$, and this is consistent with other studies

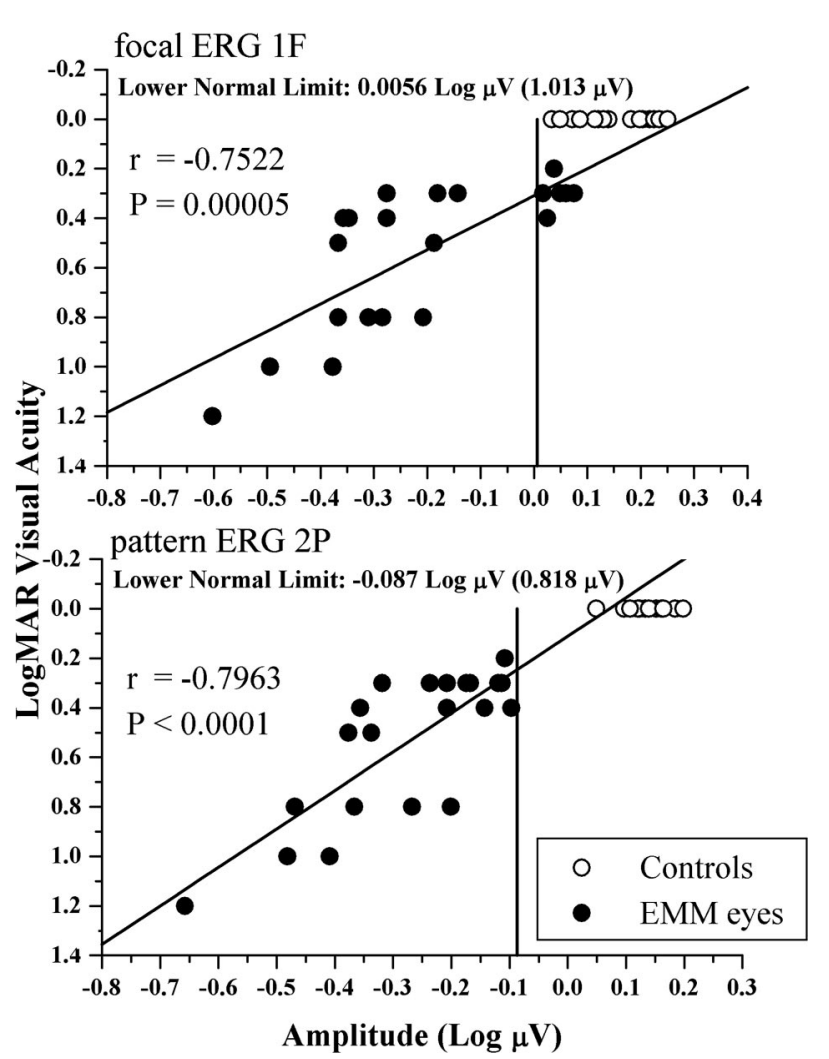

Fig. 3. At baseline, focal electroretinogram (ERG) first harmonic (1F) and pattern ERG second harmonic (2P) amplitudes observed in individual epimacular membrane (EMM) eyes and in control eyes plotted against logarithm of the minimal angle of resolution (logMAR) visual acuity. Liner regression analysis was performed by the Pearson test. Lower limit, mean values - 3 SDs for our control subjects. 


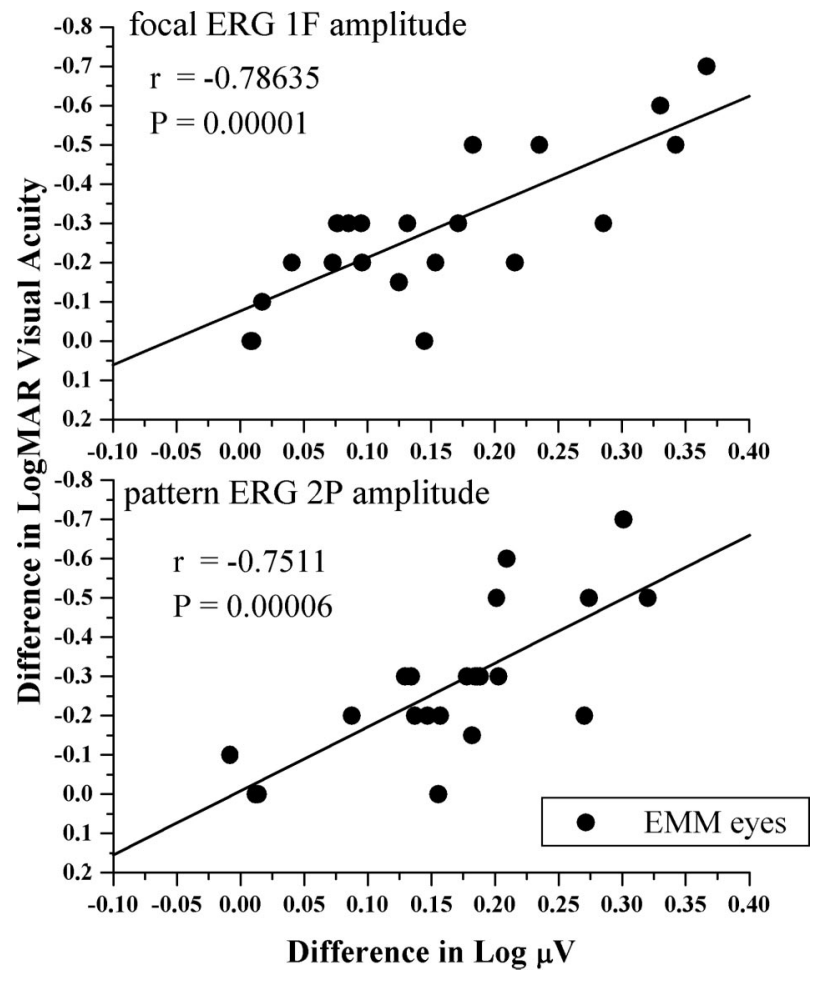

Fig. 4. Differences in focal electroretinogram (ERG) first harmonic (1F) and pattern ERG second harmonic (2P) amplitudes in individual epimacular membrane (EMM) eyes observed 6 months after surgical treatment with respect to baseline values plotted against the differences in logarithm of the minimal angle of resolution (logMAR) visual acuity. Linear regression analysis was performed by the Pearson test.

describing dysfunction of preganglionic elements in eyes affected by the same condition. ${ }^{7-9}$ In agreement with these studies, these events can be ascribed to changes in intraretinal structures leading to the formation of macular edema. ${ }^{27}$ Seven of our 22 EMM eyes had a normal fERG response, suggesting that in these cases there is possible functional sparing of the outer retinal layers (preganglionic elements). ${ }^{6}$

The presence of abnormal pERG responses in EMM eyes suggests that functional impairment is present not only in preganglionic elements but also in the innermost retinal layers (ganglion cells and their fibers) $)^{6,15,17-19}$ of the macular region. Nevertheless, in 15 EMM eyes with concomitant abnormalities of fERG and pERG responses, the observed dysfunction of the innermost macular layers (revealed by abnormal pERG) may be a primary impairment caused by epiretinal membrane, the consequence of impairment of photoreceptors, or both. On the other hand, in seven EMM eyes with normal fERG responses, the pERG impairment could be ascribed to selective dysfunction of the innermost macular layers. ${ }^{6,15}$ The pERG impairment found in our EMM eyes could also be related to the presence of changes in intraretinal structures associated with edema. Nevertheless, a possible role of the mechanical traction induced by EMM on ganglionic elements could also be considered.

These data lead us to believe that the functional impairment of preganglionic and ganglionic elements observed in EMM eyes, leading to reduction in visual acuity, may be ascribed to the presence of macular edema, mechanical effects of the epiretinal membrane, or both.

\section{Months After Surgery}

After surgical treatment, our EMM eyes had a significant increase in visual acuity, and this is in agreement with other studies reporting improvement of visual acuity after surgical removal of EMM. ${ }^{28-30}$ Improvement of visual acuity seems to be related to the postoperative anatomical recovery of macular structure (i.e., disappearance of the epiretinal tissue, reduction of the macular edema, and partial restoration of normal retinal anatomy), and this could depend on the surgical techniques used. In particular, postoperative results may be influenced by ILM removal. ${ }^{31-38}$ Nevertheless, the efficacy of ILM removal to achieve better functional results is still controversial. $^{31,35}$ In our series of EMM eyes, surgical treatment consisted of pars plana vitrectomy without intentional peeling of ILM. Because histopathological examination of the excised tissue was not performed, we cannot exclude unintentional removal of ILM in some cases, and this could represent a potential limitation of our study.

In our EMM eyes, the increase in visual acuity was related to a significant increase in fERG $1 \mathrm{~F}$ and $\mathrm{pERG}$ $2 \mathrm{P}$ amplitude responses with respect to baseline (Fig. 4). Our postoperative fERG results are in agreement with those of other electrophysiological studies describing improvement of the function of preganglionic elements (i.e., photoreceptors and amacrine cells) of the macular region in EMM eyes after surgical removal of EMM by means of fERG, multifocal electroretinogram, and oscillatory potential recordings. ${ }^{8,9}$ However, these electrophysiological techniques (i.e., fERG and multifocal electroretinogram) are not able to detect the activity of ganglion cells and their fibers, ${ }^{6,13}$ and these studies ${ }^{8,9}$ did not provide information regarding function of the innermost retinal layers after removal of EMM.

pERG was carried out in this study to provide functional evaluation of ganglion cells and their fibers, to determine the effects of surgical treatment on the innermost retinal layers. The postoperative pERG changes found in our EMM eyes could suggest that surgical removal of EMM may induce improvement 
of the function of ganglion cells and their fibers. ${ }^{6,15,18,19}$ In seven EMM eyes with abnormal pERG responses and concomitant normal function of the outer macular layers (detected by normal fERG responses at baseline), pERG improvement could be ascribed to a selective functional increase in the innermost macular layers. This improvement could be due to changes in intraretinal structures associated with reduction of edema. In addition, it could be hypothesized that EMM removal may reduce the mechanical traction induced on the innermost macular layers with consequent partial functional restoration of ganglionic elements and their fibers. In 15 EMM eyes with abnormal baseline fERG responses, it was not possible to establish if pERG improvement was a primary effect of macular membrane removal on ganglion cells and their fibers or if it was the consequence of improvement of the photoreceptor function (increase in fERG response) or both. Because increases in fERG $1 \mathrm{~F}$ and pERG $2 \mathrm{P}$ amplitudes in our EMM eyes were both significantly related to visual acuity increase (Fig. 4), it could be suggested that improvement of the function of both innermost and outer retinal layers is required to obtain improvement of visual acuity after surgical treatment.

Our results, in agreement with the results from other studies, 8,9 lead us to believe that macular preganglionic elements may have the potential to determine partial functional recovery when there is EMM peeling. Our study also suggests that the innermost macular retinal layers may have a similar potential, with consequent partial functional recovery after surgical treatment.

In two EMM eyes (EMM\#11 and EMM\#13) with unchanged visual acuity after surgery, it is possible that lack of restoration of the retinal structure with concomitant lack of functional improvement of preganglionic and ganglionic elements (unmodified fERG and pERG postoperative responses) may have occurred. In the other case (EMM\#5) with unmodified postoperative visual acuity, it can be hypothesized that the increased function of ganglionic and preganglionic elements was not sufficient to lead to improvement in visual acuity or that there was a possible lack of restoration of the retinal structure (e.g., persistence of distortion of macular elements).

In conclusion, in EMM eyes, visual acuity decrease is related to dysfunction of both preganglionic (abnormal fERG) and ganglionic (abnormal pERG) macular elements. Surgical removal of EMM, with consequent partial recovery of the macular microanatomy, could induce improvement of the function of both outer and innermost macular retinal layers, leading to a related increase in visual acuity.
Key words: epimacular membrane, focal electroretinogram, pattern electroretinogram, macular function.

\section{Acknowledgments}

The authors are grateful to Dr. Daniela Gazzaniga for her help in the selection of patients and to Banca Intesa for the support provided for this study.

\section{References}

1. Wilkins JR, Puliafito CA, Hee MR, et al. Characterization of epiretinal membranes using optical coherence tomography. Ophthalmology 1996;103:2142-2151.

2. Massin P, Aflouch C, Haouchine B, et al. Optical coherence tomography of idiopathic macular epiretinal membranes before and after surgery. Am J Ophthalmol 2000;130:732-739.

3. Azzolini C, Patelli F, Codenotti M, et al. Optical coherence tomography in idiopathic epiretinal macular membrane surgery. Eur J Ophthalmol 1999;9:206-211.

4. Wiznia RA. Natural history of idiopathic preretinal macular fibrosis. Ann Ophthalmol 1982;14:876-878.

5. Rohrschneider K, Bultmann S, Kruse FE, Volcker HE. Functional changes measured with SLO in idiopathic macular holes and in macular changes secondary to premacular fibrosis. Function in macular holes. Int Ophthalmol 2001;24:177184.

6. Parisi V, Falsini B. Electrophysiological evaluation of the macular cone system: focal electroretinography and visual evoked potentials after photostress. Semin Ophthalmol 1998; 13:178-188.

7. Tanikawa A, Horiguchi M, Kondo M, et al. Abnormal focal macular electroretinograms in eyes with idiopathic epimacular membrane. Am J Ophthalmol 1999;127:559-564.

8. Niwa T, Terasaki H, Kondo M, et al. Function and morphology of macula before and after removal of idiopathic epiretinal membrane. Invest Ophthalmol Vis Sci 2003;44:16521656.

9. Moschos M, Apostolopoulos M, Ladas J, et al. Assessment of macular function by multifocal electroretinogram before and after epimacular membrane surgery. Retina 2001;21:590_ 595.

10. Seiple WH, Siegel IM, Carr RE, Mayron C. Evaluating macular function using the focal ERG. Invest Ophthalmol Vis Sci 1986;27:1123-1130.

11. Falsini B, Fadda A, Iarossi G, et al. Retinal sensitivity to flicker modulation: reduced by early age-related maculopathy. Invest Ophthalmol Vis Sci 2000;41:1498-1506.

12. Miyake Y, Shiroyama N, Ota I, Horiguchi M. Local macular electroretinographic responses in idiopathic central serous chorioretinopathy. Am J Ophthalmol 1988;106:546-550.

13. Iarossi G, Falsini B, Piccardi M. Regional cone dysfunction in retinitis pigmentosa evaluated by flicker ERGs: relationship with perimetric sensitivity losses. Invest Ophthalmol Vis Sci 2003;44:866-874.

14. Parisi V, Canu D, Iarossi G, et al. Altered recovery of macular function after bleaching in Stargardt's disease-fundus flavimaculatus: pattern VEP evidence. Invest Ophthalmol Vis Sci 2002;43:2741-2748.

15. Varano M, Parisi V, Tedeschi M, et al. Macular function after PDT in myopic maculopathy: psychophysical and electro- 
physiological evaluation. Invest Ophthalmol Vis Sci 2005; 46:1453-1462.

16. Hood DC. Assessing retinal function with the multifocal technique. Prog Retin Eye Res 2000;19:607-646.

17. Porciatti V, Falsini B, Fadda A, Bolzani R. Steady-state analysis of the focal ERG to pattern and flicker: relationship between ERG components and retinal pathology. Clin Vis Sci 1989;4:323-332.

18. Falsini B, Bardocci A, Porciatti V, et al. Macular dysfunction in multiple sclerosis revealed by steady-state flicker and pattern ERGs. Electroencephalogr Clin Neurophysiol 1992; 82:53-59.

19. Parisi V, Pierelli F, Restuccia R, et al. VEP after photostress response in multiple sclerosis patients previously affected by optic neuritis. Electroencephalogr Clin Neurophysiol 1998; 108:73-79.

20. Gass JDM. Vitreous maculopathies. In: Gass JDM, ed. Stereoscopic Atlas of Macular Diseases. St. Louis: CV Mosby; 1987:676-713.

21. Clarkson JG, Green WR, Massof D. A histopathologic review of 168 cases of preretinal membranes. Am J Ophthalmol 1977;84:1-17.

22. Michels RG. A clinical and histopathologic study of epiretinal membranes affecting the macula and removed by vitreous surgery. Trans Am Ophthalmol Soc 1982;80:580-656.

23. Smiddy WE, Maguire AM, Green WR, et al. Idiopathic epiretinal membranes: ultrastructural characteristics and clinicopathologic correlation. Ophthalmology 1989;96:811-820.

24. Smiddy WE, Michel RG, Green WR. Morphology, pathology and surgery of idiopathic vitreoretinal macular disorders. Retina 1990;10:288-296.

25. Wallow IH, Stevens TS, Greaser ML, et al. Actin filaments in contracting preretinal membranes. Arch Ophthalmol 1984; 102:1370-1375.

26. Puliafito C, Hee M, Lin C, et al. Imaging of macular diseases with optical coherence tomography. Ophthalmology 1995; 102:217-229.
27. Miyake Y, Miyake K, Shiroyama N. Classification of aphakic cystoid macular edema with focal macular electroretinograms. Am J Ophthalmol 1993;116:576-583.

28. Kwok AK, Lai TY, Yuen KS. Epiretinal membrane surgery with or without internal limiting membrane peeling. Clin Experiment Ophthalmol 2005;33:379-385.

29. Wong JG, Sachdev N, Beaumont PE, Chang AA. Visual outcomes following vitrectomy and peeling of epiretinal membrane. Clin Experiment Ophthalmol 2005;33:373-378.

30. Hillenkamp J, Saikia P, Gora F, et al. Macular function and morphology after peeling of idiopathic epiretinal membrane with and without the assistance of indocyanine green. Br J Ophthalmol 2005;89:437-443.

31. Sivalingam A, Eagle RC Jr, Duker JS, et al. Visual prognosis correlated with the presence of internal-limiting membrane in histopathologic specimens obtained from epiretinal membrane surgery. Ophthalmology 1990;97:1549-1552.

32. Michels RG. Vitrectomy for macular pucker. Ophthalmology 1984;91:1384-1388.

33. Poliner LS, Olk RJ, Grand MG, et al. Surgical management of premacular fibroplasia. Arch Ophthalmol 1988;106:761764.

34. de Bustros S, Thompson JT, Michels RG, et al. Vitrectomy for idiopathic epiretinal membranes causing macular pucker. Br J Ophthalmol 1988;72:692-695.

35. Koerner F, Garweg J. Vitrectomy for macular pucker and vitreomacular traction syndrome. Doc Ophthalmol 1999;97: 449-458.

36. Donati G, Kapetanios AD, Pournaras CJ. Surgery of idiopathic epiretinal membranes: functional results and complications. J Fr Ophtalmol 1998;21:654-660.

37. Von Gunten S, Pournaras CJ, de Gottrau P, Brazitikos P. Prognostic factors in surgical treatment of epiretinal membranes. Klin Monatsbl Augenheilkd 1994;204:309-312.

38. Park DW, Dugel PU, Garda J, et al. Macular pucker removal with and without internal limiting membrane peeling: pilot study. Ophthalmology 2003;110:62-64. 